\title{
Short communication \\ Sulphate measurement in organic-rich solutions: Carbonate fusion pretreatment to remove organic interferences
}

\author{
NE Ristow, SW Sötemann, MC Wentzel*, RE Loewenthal and GA Ekama \\ Water Research Group, Department of Civil Engineering, University of Cape Town, Rondebosch 7701, South Africa
}

\begin{abstract}
Sulphate measurement using a barium sulphate turbidimetric method in solutions with high concentrations of organic material is shown to be problematic. The organics give background colour, which introduces a positive error to the measured absorption, and inhibit the barium sulphate precipitate, which results in a negative error. A carbonate fusion pretreatment of the sample results in the removal of the organic matter and associated interferences. With this pretreatment, excellent sulphate recoveries were obtained $(100 \%)$. Rigorous testing of the method shows that reproducible and accurate results are obtainable.
\end{abstract}

Keywords: sulphate measurement, organic interference, carbonate fusion

\section{Introduction}

In a variety of applications of biological sulphate reduction, measurement of sulphate in the presence of elevated concentrations of organic materials is required. Such measurements have proved problematic due to interferences caused by the organic material. This paper investigates the interference of organics in sulphate measurement by standard methods, and proposes modifications to the prescribed methods to overcome these interferences.

Standard Methods (1985) describes four methods for the determination of sulphate in water: Methods 426A, B, C and D Two of these methods (426A and 426B) involve the formation of a barium sulphate precipitate in hydrochloric acid at near boiling temperature, followed by a period of digestion $(>2 \mathrm{~h})$, filtration of the barium sulphate, which is then either dried (426B) or ignited (426A), and the residue weighed. However, both methods are described as being subject to much interference, leading to both positive and negative errors. Interferences include suspended matter, silica, barium chloride, nitrate, sulphide and alkali metals, all of which add to the mass of the dried or ignited residue, or substitute the barium ion with one of a lower or higher molecular weight. Accordingly, these two test methods were rejected for further evaluation.

Method 426D requires the availability of an auto-analyser, which would render it unfeasible unless large quantities of samples were being tested and hence was not investigated further. Method 426C involves the formation of a barium sulphate precipitate in an acetic acid buffer solution, and the measurement of the absorbance of the precipitate using a spectrophotometer at $420 \mathrm{~nm}$ wavelength. However, Standard Methods (1985) lists colour, suspended matter and organic material as the major interferences. If the organic material and colour can be removed from the sample to allow for the accurate measurement of the sulphate concentration, the method would seem practical and

\footnotetext{
* To whom all correspondence should be addressed. III +2721 650-2583; fax: +2721 689-7471; e-mail:markw@ebe.uct.ac.za Received 1 November 2004.
}

feasible. Accordingly, a preliminary evaluation of the method was undertaken.

\section{Preliminary evaluation}

Method 426C can be used automated with an auto-analyser, and the accuracy of this automated method was evaluated and also compared with a commercially available sulphate test kit (Merck, Method No 14791). A standard sulphate solution $\left(100 \mathrm{mgSO}_{4} / \ell\right)$ was prepared and increasing amounts of sulphate-free soluble organic solution (soluble fraction of methanogenic anaerobic digester effluent, filtered through a $0.45 \mu \mathrm{m}$ filter paper, S\&S ME 25/21) were added to the standard solution and the sulphate concentration measured using both methods, see Table 1. Clearly, for both methods, as the organic concentration in the sample increases, the accuracy of the analysis deteriorates. The accuracy of the test kit was inferior both for the solutions without sulphate present and for the standard sulphate solution without organic matter, and accordingly the test kit version of the method was discarded.

\begin{tabular}{|c|c|c|c|}
\hline \multicolumn{4}{|c|}{$\begin{array}{c}\text { TABLE } 1 \\
\text { Sulphate concentrations of two experiments to } \\
\text { determine the effects of a sulphate free soluble } \\
\text { organic solution on the analysis of a standard } \\
\text { sulphate solution }\left(100 \mathrm{mgSO}_{4} / \ell\right)\end{array}$} \\
\hline \multirow{2}{*}{$\begin{array}{l}\text { Sulphate } \\
\text { concen- } \\
\text { tration } \\
\left(\mathrm{mgSO}_{4} / \mathrm{l}\right)\end{array}$} & \multirow{2}{*}{$\begin{array}{c}\text { Volume } \\
\text { organics } \\
\text { added } \\
(\mathrm{m} l)\end{array}$} & \multicolumn{2}{|c|}{ Sulphate concentration $\left(\mathrm{mgSO}_{4} / \mathrm{l}\right)$} \\
\hline & & Merck method & Auto-analyser \\
\hline 0 & 10 & $112 ; 83 ; 42$ & $0 ; 0 ; 0$ \\
\hline 0 & 20 & $77 ; 66 ; 78$ & $5 ; 0 ; 0$ \\
\hline 100 & 0 & $134 ; 149 ; 130$ & $103 ; 101 ; 100$ \\
\hline 100 & 10 & $108 ; 132 ; 152$ & $112 ; 113 ; 112$ \\
\hline 100 & 20 & $119 ; 123 ; 120$ & $138 ; 138 ; 18$ \\
\hline
\end{tabular}

Although Table 1 clearly illustrates the interference of soluble organic matter on the accuracy of Method $426 \mathrm{C}$ using an auto-analyser, the accuracy of the method for samples without organic interference was encouraging. Also, the method shows 
that the organic solution added to the sulphate standard solution was indeed sulphate-free (of importance in the experiments that follow). Accordingly, the method was selected for modification, to develop pretreatments to remove the organics prior to application.

\section{Method development}

As noted above, Standard Methods (1985) lists colour, suspended matter and organic material as the major interferences in Method 426C. The method is based on the formation of a barium sulphate $\left(\mathrm{BaSO}_{4}\right)$ precipitate of uniform size under controlled $\mathrm{pH}$ conditions using an acetate buffer, and then measuring the absorbance of the $\mathrm{BaSO}_{4}$ suspension using a spectrophotometer. Filtration of the sample will remove the suspended matter and particulate organic material, especially if used in conjunction with a flocculent, and hence these interferences can be readily overcome. However, interference by the colour of the sample, and particularly soluble organics, would be problematic, and need to be addressed.

\section{Calibration curve}

The turbidimetric method (Method 426C) requires the generation of a standard calibration curve. To overcome the colour interference (and the soluble organic interference if this is due to colour only), the standard sulphate samples could be measured in the presence of the same, or similar, background matrix as the sulphate samples, so that the colour interferences would be incorporated into the calibration curve. Comparison of the calibration curve with one in which the background matrix is distilled water would also allow the magnitude of the interferences to be observed. Thus, a calibration curve was generated using a standard sulphate solution to which different amounts of methanogenic anaerobic digestion filtered effluent, in which the sulphate concentration would be zero, was added (similar to the experiments of Table 1).

The calibration curves generated in this manner were inconsistent. The addition of a fixed volume of organic matter to varying sulphate concentrations resulted in a consistent curve, but this curve was not reproducible. Further, when the organic addition was either halved or doubled, the curves generated gave no correlation to the amount of organic addition. Thus, the generation of a general calibration curve in the presence of the background organic matrix was not possible, nor would it be possible to use a series of calibration curves at different organic concentrations if the organic concentration of the sample was known. Thus, it was concluded that it is imperative that the organic background be removed from the sample prior to sulphate analysis.

To remove the organics from the sample, several alternatives were investigated but did not prove feasible. For example, with dichromate and nitric acid pre-digestion, the colour of the dichromate gave an absorbance greater than the barium sulphate precipitate. However, one method did appear promising, that of carbonate fusion, and this was investigated further. This carbonate fusion method proved very successful for removal of colour interference in measurement of total phosphorus in municipal wastewater (Martin and Marais, 1975).

\section{Organic interference removal by carbonate fusion}

Prior to sulphate analysis (Method 426C above), the sample was to be passed through a carbonate fusion pretreatment, which would oxidise the organics and possibly remove both the colour and organic interferences. The carbonate fusion method involved drying a measured volume $(10 \mathrm{~m} \ell)$ of the filtered sample $(0.45 \mu \mathrm{m}$ membrane filter $)$ in a crucible. Both platinum and nickel crucibles were used without any difference in the results. Drying was achieved using either a steam bath or an oven. The advantage of using an oven was that the samples could be left unattended, while the steam bath required supervision. However, if the temperature of the oven was too high $\left(>150^{\circ} \mathrm{C}\right)$, the dried residue tended to splatter, and part of the sample was lost from the crucible.

Once dry, a half-teaspoon of sodium carbonate $\left(\mathrm{Na}_{2} \mathrm{CO}_{3}\right)$ was added, and the crucible heated over a powerful Bunsen burner until the sodium carbonate melted, forming a sodium meta-sulphate salt. This took around $30 \mathrm{~s}$ on a powerful burner, or several minutes on a normal Bunsen burner. Once the sodium carbonate had melted, the molten contents were swirled so that it came into contact with the entire initial sample. This was then allowed to cool. The cooled sodium meta-sulphate salt was then dissolved by adding sufficient $(\sim 10 \mathrm{~m} \ell, 1: 1)$ concentrated $\mathrm{HCl}$ solution $(12 \mathrm{~N})$ via a pipette. The acid reacted vigorously with the salt as carbon dioxide was expelled, so that a watch glass was required to cover the crucible to prevent loss of sample. Once the sample ceased fizzing, the crucible contents were poured into a $50 \mathrm{~m} \ell$ or $100 \mathrm{~m} \ell$ volumetric flask, depending on the dilution

\begin{tabular}{|c|c|c|c|}
\hline \multicolumn{4}{|c|}{$\begin{array}{c}\text { TABLE } 2 \\
\text { Values for the absorbencies }(420 \mathrm{~nm}) \text { obtained from standard sulphate concentra- } \\
\text { tions at difference concentrations of background organics, all pretreated by the } \\
\text { carbonate fusion method }\end{array}$} \\
\hline $\begin{array}{l}\mathrm{SO}_{4} \text { concentration } \\
\left(\mathrm{mg} \mathrm{SO}_{4} / \ell\right)\end{array}$ & $\begin{array}{l}\text { Absorption with x50 } \\
\text { organic dilution }\end{array}$ & $\begin{array}{l}\text { Absorption with } \times 100 \\
\text { organic dilution }\end{array}$ & $\begin{array}{l}\text { Absorption with } \times 200 \\
\text { organic dilution }\end{array}$ \\
\hline 0 (blank) & 0.000 & 0.000 & 0.000 \\
\hline 10 & 0.070 & $0.064,0.070$ & $0.070,0.065$ \\
\hline 15 & & $0.123,0.123$ & \\
\hline 20 & $0.170,0.178$ & $0.170,0.170$ & $0.176,0.171$ \\
\hline 25 & & $0.220,0.240$ & \\
\hline 30 & 0.298 & $0.316,0.303$ & $0.313,0.314$ \\
\hline 35 & & $0.352,0.374$ & \\
\hline 40 & $0.428,0.428$ & $0.410,0.430$ & $0.380,0.382$ \\
\hline 50 & 0.572 & $0.555,0.570$ & 0.558 \\
\hline
\end{tabular}


required for the final sample. The crucible and cover glass were rinsed with distilled water and the rinse water captured into the volumetric flask. A drop of phenolphthalein indicator was added to the volumetric flask, followed by $10 \mathrm{M}$ sodium hydroxide solution (drop wise) until the solution turned pink. The flask was then made up to volume with distilled water. This solution was then further diluted into $50 \mathrm{~m} \ell$ volumetric flasks so that the final concentration was in the recommended range of 10 to 40 $\mathrm{mgSO}_{4} / \ell$. The diluted sample then was subjected to the standard sulphate measurement method, Method 426C.

\section{Carbonate fusion calibration curve}

A calibration curve was generated by first making up standard sulphate solutions with the methanogenic organic sample as background. These standards were then pretreated using the carbonate fusion method above, and then subjected to the sulphate measurement Method 426C. Two further calibration curves were generated in the same way, but with different dilutions of the same methanogenic organic sample as background. Table 2 lists the absorbance values for the three calibration curves, while Fig. 1 plots these calibration curves.

From Table 2 and Fig. 1, there is little difference between the absorbences from the standard curves with the three different organic concentrations. Clearly, the carbonate fusion pretreatment of the samples successfully removed all organic interferences. This allows for a single calibration curve to be used for all sample initial organic concentrations and background matrices.

Standard Methods (1985) states that the $\mathrm{BaSO}_{4}$ turbidity method $426 \mathrm{C}$ is restricted to the range of 10 to $40 \mathrm{mgSO}_{4} / \ell$. In the study here, although the absorbance at zero sulphate concentration is zero, the relationship between the sulphate concentration and the absorbance is non-linear between 0 and $10 \mathrm{mgSO}_{4} / \ell$ (Fig. 1), confirming the lower measurement limit. As expected, the relationship is linear between 10 and $40 \mathrm{mgSO}_{4} / \ell$, but the linearity continues above $40 \mathrm{mgSO}_{4} / \ell$, suggesting a higher upper limit of at least $50 \mathrm{mgSO}_{4} / \ell$. This possibly could be extended further, but was not evaluated here.

To test whether standard samples used to generate the calibration curve require carbonate fusion pretreatment or not, a calibration curve was generated using a standard sulphate solution and deionised water only. A sample of this standard sulphate solution was put through the carbonate fusion pretreatment, and the absorbance compared to the calibration curve. The $120 \mathrm{mgSO}_{4} / \ell$ sample was analyzed at 119 and $122 \mathrm{mgSO}_{4} / \ell$ (99.17 and $101.17 \%$ respectively). Thus, irrespective of whether the standard samples in the calibration curve are pretreated with carbonate fusion or not, the same absorbance is obtained. This indicates that the carbonate fusion pretreatment does not impact the subsequent sulphate measurement method (Method 426C). Hence, a standard curve using deionised water will suffice for samples that are being treated by the carbonate fusion method.

\section{Method verification}

To verify the proposed method, two samples (S1 and S2) were drawn from two laboratory-scale anaerobic digesters (one from each) being fed a mixture of primary sewage sludge (PSS) and sulphate. A third sample (M1) was drawn from a purely methanogenic laboratory-scale anaerobic digester also treating PSS. A few grains of zinc acetate ( $\mathrm{ZnAc}$ ) were added to the two samples (S1 and S2) (sulphate-reducing systems) to precipitate any sulphide present, since these systems generate sulphide: aqueous sulphide would be oxidised to sulphate in the carbonate fusion

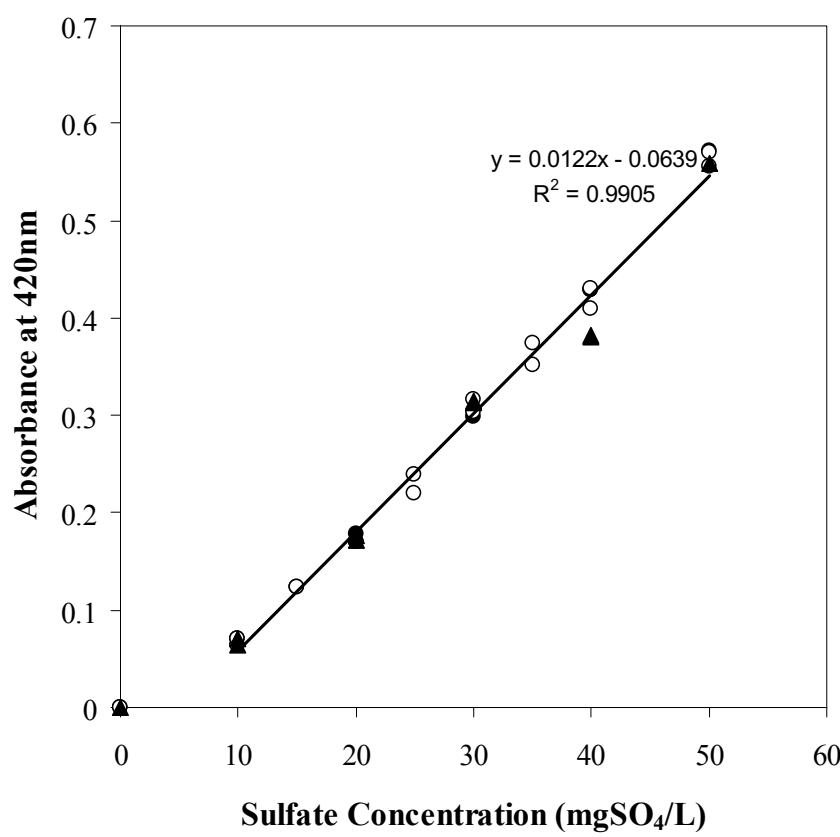

- $\mathrm{x} 50 \circ \mathrm{x} 100 \Delta \mathrm{\Delta} 200$

Figure 1

Sulphate calibration curves with three different background organic concentrations; all samples pretreated with the carbonate fusion method

pretreatment step and hence add to the sulphate measured in the subsequent $\mathrm{BaSO}_{4}$ turbidity step. $\mathrm{NaOH}$ was added to precipitate the residual $\mathrm{Zn}$ as $\mathrm{Zn}(\mathrm{OH})_{2}$, which also acted as a flocculent. The samples were settled for $1 \mathrm{~min}$, and the supernatant then filtered through a $0.45 \mu \mathrm{m}$ membrane filter paper (S\&S ME25/21). The filtrate samples were used for a series of experiments to demonstrate the effects of the background matrix on the sulphate analysis. Sample S1 and S2 $0.45 \mu \mathrm{m}$ filtrates were analyzed directly, but with appropriate dilution with distilled water to bring the sulphate concentrations into the measurement range (see above), Table 3. A third sample (S2 and M1) was made up from a mixture of sample S2 and M1 filtrates, with the addition of M1 to S2 to give the same dilution as for S2 only above. It is important to note that the methanogenic anaerobic digester from which sample M1 was drawn was operating under very stable conditions, and thus the VFA concentration was negligible. However, sample M1 was visibly brown in colour.

The three samples (S1, S2 and S2 + M1) were divided into two duplicates. The one set of samples had no pretreatment, while the other set was subjected to pretreatment using the carbonate fusion method above. The absorbences of the three blank samples (S1, S2 and S2 + M1) were measured, without barium chloride addition, for both the non-treated and carbonate-fusion pretreated sets of samples. Each sample in both sets was then divided into two and the $\mathrm{BaSO}_{4}$ turbidity method (426C) applied to the duplicates, and the absorbance measured. Absorbences were converted to "equivalent" sulphate concentrations with the calibration curve in Fig. 1, and the dilution taken into account, see Table 3.

From Table 3, it is clear that the presence of the organic solution significantly affects the measurement of the sulphate. For the sample set without carbonate fusion pretreatment, the colour in the blanks results in significant absorbences, which translated to significant sulphate concentrations ( 83 to $135 \mathrm{mgSO}_{4} / \ell$ ). 


\begin{tabular}{|c|c|c|c|}
\hline \multicolumn{4}{|c|}{$\begin{array}{c}\text { TABLE } 3 \\
\text { Equivalent sulphate concentrations for samples } \mathrm{S} 1, \mathrm{~S} 2 \text { and } \mathrm{S} 2+\mathrm{M} 1 \text {, with } \\
\text { and without organic and barium chloride }\left(\mathrm{BaCl}_{2}\right) \text { addition, and with and } \\
\text { without carbonate fusion pretreatment }\end{array}$} \\
\hline \multirow[t]{2}{*}{ Treatment } & \multicolumn{3}{|c|}{ Sulphate Concentration $\left(\mathrm{mgSO}_{4} / \mathrm{l}\right)$} \\
\hline & $\begin{array}{c}\text { S1 } \\
\text { Sulphate } \\
\text { Sample 1 }\end{array}$ & $\begin{array}{c}\text { S2 } \\
\text { Sulphate } \\
\text { Sample 2 }\end{array}$ & $\begin{array}{c}\text { S2+M1 } \\
\text { Sulphate } \\
\text { sample } \\
+ \text { soluble } \\
\text { organic } \\
\text { solution } \\
\end{array}$ \\
\hline Dilution & 20 & 6.66 & 6.66 \\
\hline Blank (no $\mathrm{BaCl}_{2}$; no carbonate fusion) & 135 & 83 & 93 \\
\hline $\mathrm{BaCl}_{2}$ added; no carbonate fusion & $246 ; 352$ & $85 ; 85$ & $96 ; 97$ \\
\hline Blank (no $\mathrm{BaCl}_{2}$; carbonate fusion) & 0 & 0 & 0 \\
\hline Carbonate fusion; $\mathrm{BaCl}_{2}$ added & $276 ; 262$ & $99 ; 99$ & $98 ; 98$ \\
\hline
\end{tabular}

Method 426C suggests that the absorbance of the blank sample be subtracted from the absorbance of the sample to which the barium chloride has been added. In this case, for sample S2, the calculated sulphate concentration would be $2 \mathrm{mg} \mathrm{SO}_{4} / \ell(85$ - $83 \mathrm{mgSO}_{4} / \ell$ ), and when the organic solution (M1) was added, it would increase to 3 to $4 \mathrm{mgSO}_{4} / \ell$ ( $96-97$ to $93 \mathrm{mgSO}_{4} / \ell$ ). For sample S1, the difference between the two duplicate measured concentrations ( 246 and $352 \mathrm{mgSO}_{4} / \ell$ ) is so great that an accurate estimate is not possible. However, for all three samples, the carbonate fusion pretreatment successfully removed all colour interferences, in that no absorbance and hence sulphate concentrations were measured for the blank samples. Further, the measured concentrations were more consistent, 276 and $262 \mathrm{mgSO}_{4} / \ell$ for S1, 99 and 99 and 98 and $98 \mathrm{mgSO}_{4} / \ell$ for S2 with and without M1 addition respectively. Additionally, a third S1 sample at a different dilution was analyzed including carbonate fusion pretreatment, and gave a concentration of $288 \mathrm{mgSO}_{4} / \ell$ (compared with 276 and $262 \mathrm{mgSO}_{4} / \ell$, Table 3 ). With the carbonate fusion pretreatment, the sulphate concentration in sample S1 (98 to 99 $\left.\mathrm{mgSO}_{4} / \ell\right)$ is significantly higher than that obtained without the pretreatment ( 2 to $4 \mathrm{mgSO}_{4} / \ell$ ). This, and the change in sulphate concentration induced by adding organics would suggest that the organics interference in the test method is not only background colour, but also in the $\mathrm{BaSO}_{4}$ precipitate. However, since the actual sulphate concentration in the samples is not known, it cannot be stated unequivocally that the carbonate fusion pretreatment measurement is superior. Accordingly, sulphate recovery tests were undertaken.

Following the procedures above, a standard sulphate solution was added to a sulphate-reducing system sample (similar to $\mathrm{S} 1$ above) with unknown sulphate concentration. Additions were made to the equivalent of 0,20 and $40 \mathrm{mgSO}_{4} / \ell$, and the respective measured concentrations were 44,64 and $84 \mathrm{mgSO}_{4} / \ell$, indicating a $100 \%$ sulphate recovery. This substantially verifies the carbonate fusion pretreatment modification to the test method (Method 426C).

To test the reproducibility of the carbonate fusion pretreatment method, four samples were drawn separately from a single anaerobic digester being fed a sulphate and primary sewage sludge (PSS) mixture and analyzed following the procedures de- tailed above. The results for the four samples were 129, 122, 119 and $124 \mathrm{mgSO}_{4} / \ell$, showing excellent reproducibility (coefficient of variation $=0.03$ ).

To further demonstrate the interference of organics in the method, a soluble organic solution (methanogenic supernatant, brown in colour, no sulphate, see above) was added to the samples after the carbonate fusion pretreatment step, but before the $\mathrm{BaSO}_{4}$ precipitation. The results showed that the addition of 5 and $10 \mathrm{~m} \ell$ of methanogenic organic solution (no $\mathrm{SO}_{4}$ ) gave an increase in the equivalent measured sulphate concentration (128 $\mathrm{mgSO}_{4} / \ell$ (no organic); $142 \mathrm{mgSO}_{4} / \ell\left(5 \mathrm{~m} \ell\right.$ organic); $170 \mathrm{mgSO}_{4} / \ell$ $(10 \mathrm{~m} \ell$ organic) $)$. This re-enforces the interferences in measuring sulphate in high organic solutions.

\section{Conclusions}

The presence of organic matter interferes with the $\mathrm{BaSO}_{4}$ turbidimetric method (Method 426C, Standard Methods, 1985) for the determination of sulphate. The interference of the organics in the method is due both to their background colour, and to their interference in the $\mathrm{BaSO}_{4}$ precipitation. Carbonate fusion pretreatment has been shown to successfully remove these interferences, and excellent recoveries of added sulphate were obtained. The detailed method is available from the authors.

\section{Acknowledgements}

This research was supported by the Water Research Commission, National Research Foundation and the University of Cape Town, and is published with their permission.

\section{References}

MARTIN KAC and MARAIS GvR (1975) Kinetics of enhanced phosphorus removal in the activated sludge process. Research Report No. W14, Department of Civil Engineering, University of Cape Town, Rondebosch, 7701, South Africa.

STANDARD METHODS (1985) Standard Methods for the Examination of Water and Wastewater (1985) (19th edn.). American Public Health Association/American Water Works Association/Water Environment Federation, Washington DC, USA. 